\title{
The Set SFmBDR of Fuzzy Time Series Forecasting Models
}

\author{
Hao Feng ${ }^{1}$, Hongxu Wang ${ }^{2}$, Chengguo $\mathrm{Yin}^{3}$ and Xiaoli $\mathrm{Lu}^{2}$ \\ ${ }^{1}$ College of Marine Science and Technology, Hainan Tropical Ocean University, Hainan Saya, China \\ ${ }^{2}$ Commercial College, Hainan Tropical Ocean University, Hainan Saya, China \\ ${ }^{3}$ College of Computer Science and Engineering, Hainan Tropical Ocean University, Hainan Saya, China
}

\begin{abstract}
Fuzzy time series forecasting models are created by Song and Chissom in 1993. In 2012, Saxena, Sharma\& Easo put forward the forecasting model which more improve the forecasting accuracy. According to this, this paper improve the set SFmBDR of fuzzy time series forecasting model based on differential rate. The forecasting model SFmBDR $(0.000002,0.000004)$ and SFmBDR $(0.000004,0.000002)$ of SFmBDR can gain the AFER $=0 \%$ and MSE $=0$, during we study the problems of enrollments data of University of Alabama in 1971 1992. They should have better application potential.
\end{abstract}

Keywords-forecasting method of fuzzy time series; fuzzy function of SFmBDR;Inverse fuzzy function of SFmBDR; forecasting function of SFmBDR

\section{INTRODUCTION}

Song and Chissom firstly come up with the fuzzy time series forecasting model and discuss the problems of enrollments data of University of Alabama in 1971 1992, which apply fuzzy set series[3]. Sequentially, they put forward many fuzzy times series forecasting model which can see in the references [1,2,4-6,8-17]. However, the forecasting accuracy is not high, and this problems are still puzzling people.Saxena, Sharma, Easo[9] (2012) propose the new set of forecasting fuzzy model. It gain the unprecedented forecasting accuracy, when they study the problems of enrollments data of University of Alabama. Therefore, it cause the researches of Wang, Guo, Feng, Jin, $\mathrm{Wu}[5,6,8,10-12,15-17]$, and they respectively present forecasting model, which have some improvement in the forecasting accuracy. This paper further excavate their research achievement, and propose the set SFmBDR of fuzzy time series forecasting model based on order differential rate. The forecasting model of SFmBDR(0.000002,0.000004) and SFmBDR(0.000004,0.000002) gain the enrollments forecasting data $\mathrm{AFER}=0 \%$ and $\mathrm{MSE}=0$, when study the problems of enrollments data of University of Alabama in 1971 1992. It achieve the highest level of forecasting accuracy.

\section{THE SET SFMBDR OF FUZZY TIME SERIES FORECASTING MODEL}

The relative concepts see the references Saxena, Sharma, Easo[9]. When we study a problem of time series forecasting, we can assume its universe of historical data as $N=\left\{N_{1}, N_{2}, \cdots\right.$, $N_{n}$ \}.The formulas of differential rate of historical data is $M_{t}$ $=\left(\mathrm{N}_{\mathrm{t}}-\mathrm{N}_{\mathrm{t}-1}\right) / \mathrm{N}_{\mathrm{t}-1}$. The universe differential rate of historical data is $M=\left\{M_{2}, M_{3}, \ldots, M_{n}\right\}$. We propose the definition of fuzzy function and inverse fuzzy function and forecasting function by spread the theories of Saxena, Sharma, Easo[9].

Definition 1: If have a time series forecasting problem, its universe of discourse of historical data as $\mathrm{N}=\left\{\mathrm{N}_{1}, \mathrm{~N}_{2}, \cdots, \mathrm{N}_{\mathrm{n}}\right\}$ and the universe differential rate of historical data is $M=\left\{M_{2}, M_{3}, \ldots, M_{n}\right\}$. If in $M$,we can be defined fuzzy series as $\mathrm{U}_{\mathrm{t}}(0.2,0.8)$ :

$$
U_{t}(0.2,0.8)=\left\{\begin{array}{l}
\frac{1}{M_{2}}+\frac{0.8}{M_{3}}+\frac{0}{M_{3}}+\cdots+\frac{0}{M_{n}}, t=2, \\
\frac{0}{M_{2}}+\cdots+\frac{0}{M_{t-2}}+\frac{0.2}{M_{t-1}}+\frac{1}{M_{t}}, 3 \leq t \leq n .
\end{array}\right.
$$

Where the inverse fuzzy series of fuzzy series $U_{t}(0.2,0.8)$ can be defined $\mathrm{S}_{\mathrm{t}}(0.2,0.8)$ :

$$
S_{t}(0.2,0.8)=\left\{\begin{array}{l}
\frac{1+0.8}{\frac{1}{M_{2}}+\frac{0.8}{M_{3}}+\frac{0}{M_{4}}+\cdots+\frac{0}{M_{n}}}, t=2, \\
\frac{0.2+1}{\frac{0}{M_{2}}+\cdots+\frac{0}{M_{t-2}}+\frac{0.2}{M_{t-1}}+\frac{1}{M_{t}}}, 3 \leq t \leq n .
\end{array}\right.
$$

Definition 2: If have a time series forecasting problem, its universe of discourse of historical data as $N=\left\{N_{1}, N_{2}, \ldots, N_{n}\right\}$ and the universe differential rate of historical data is $M=\left\{M_{2}, M_{3}, \ldots, M_{n}\right\}$. If in $M$, we can be defined inverse fuzzy series function as $U_{t}\left(\mu_{1}, \mu_{2}\right)$

$$
U_{t}\left(\mu_{1}, \mu_{2}\right)= \begin{cases}\frac{1}{M_{2}}+\frac{\mu_{2}}{M_{3}}, & \text { if } t=2 \\ \frac{\mu_{1}}{M_{t-1}}+\frac{1}{M_{t}}, & \text { if } 3 \leq t \leq n .\end{cases}
$$

Where independent variable $\quad \mu_{1} \in[0,1)$ and $\mu_{2} \in[0,1)$ also call membership of $U_{t}\left(\mu_{1}, \mu_{2}\right)$. when $\mu_{1}=\mu_{2}=\mu$, the fuzzy function SFmBDR denoted by $U_{t}(\mu) \quad(\mathrm{t}=2,3, \ldots, \mathrm{n})$. 
Definition 3: If have a time series forecasting problem, its universe of discourse of historical data as $N=\left\{N_{1}, N_{2}, \cdots, N_{n}\right\}$ and the universe differential rate of historical data is $M=\left\{M_{2}, M_{3}, \ldots, M_{n}\right\}$. If in $M$,we can be defined fuzzy series function $U_{t}\left(\mu_{1}, \mu_{2}\right)$ of inverse fuzzy function $S_{t}\left(\mu_{1}, \mu_{2}\right)$ :

$$
S_{t}\left(\mu_{1}, \mu_{2}\right)=\left\{\begin{array}{lc}
\frac{1+\mu_{2}}{\frac{1}{M_{2}}+\frac{\mu_{2}}{M_{3}}}, & \text { if } t=2, \\
\frac{\mu_{1}+1}{\frac{\mu_{1}}{M_{t-1}}+\frac{1}{M_{t}}}, & \text { if } 3 \leq t \leq n .
\end{array}\right.
$$

Where independent variable $\quad \mu_{1} \in[0,1)$ and $\mu_{2} \in[0,1)$ also call membership of $S_{t}\left(\mu_{1}, \mu_{2}\right)$. when $\mu_{1}=\mu_{2}=\mu$, the inverse fuzzy function SFmBDR denoted by $\mathrm{S}_{\mathrm{t}}(\mu)(\mathrm{t}=2,3, \ldots, \mathrm{n})$.

Definition 4: If have a time series forecasting problem, the universe differential rate of historical data is $M=\left\{M_{2}, M_{3}, \ldots\right.$, $\left.M_{n}\right\}$. As every $t \in\{2,3, \cdots, n\}$, defined forecasting function

$\mathrm{R}_{\mathrm{t}}\left(\mu_{1}, \mu_{2},\right):$

$$
R_{t}\left(\mu_{1}, \mu_{2}\right)=N_{t-1}\left(1+S_{t}\left(\mu_{1}, \mu_{2}\right)\right)
$$

Where independent variable $\mu_{1} \in[0,1)$ and $\mu_{2} \in[0,1)$ also call membership of forecasting function $\mathrm{R}_{\mathrm{t}}\left(\mu_{1}, \mu_{2}\right)$. $\mathrm{N}_{\mathrm{t}-1}$ is the $\mathrm{t}-1$ years' historical data, $\mathrm{S}_{\mathrm{t}}\left(\mu_{1}, \mu_{2}\right)$ is t years' inverse fuzzy function. when $\mu_{1}=\mu_{2}=\mu$, the inverse fuzzy function SFmBDR denoted by

$\mathrm{R}_{\mathrm{t}}(\mu)(\mathrm{t}=2,3, \ldots, \mathrm{n})$. When $\mu_{1}$ and $\mu_{2}$ get the specific membership in [0,1), $\mathrm{R}_{\mathrm{t}}\left(\mu_{1}, \mu_{2}\right)$ call the forecasting formula of SFmBDR, it can also denoted by $\operatorname{SFmBDR}\left(\mu_{1}, \mu_{2}\right)$.

If have a time series forecasting problem, its universe of discourse of historical data as $\mathrm{N}=\left\{\mathrm{N}_{1}, \mathrm{~N}_{2}, \cdots, \mathrm{N}_{\mathrm{n}}\right\}$ and the universe differential rate of historical data is $M=\left\{M_{2}, M_{3}, \ldots\right.$, $\left.\mathrm{M}_{\mathrm{n}}\right\}$. If the membership $\mu_{1}$ and $\mu_{2}$ can certain specific figure in [0,1), we can build a forecasting model $\operatorname{SFmBDR}\left(\mu_{1}, \mu_{2}\right)$ through the formula (3), we can get the following step:

First, listing the historical sheet of time forecasting problem. Second, listing the universe of discourse historical rate $\mathrm{N}$ and the universe of discourse of differential rate $\mathrm{M}$. Third, listing the forecasting formula $\operatorname{SFmBDR}\left(\mu_{1}, \mu_{2}\right)$. Fourth, applying SFmBDR $\left(\mu_{1}, \mu_{2}\right)$ to calculate the forecasting value of historical rate. Forecasting formula SFmBDR $\left(\mu_{1}, \mu_{2}\right)$ is the forecasting model SFmBDR $\left(\mu_{1}, \mu_{2}\right)$, because we follow the route: fuzzy function
$\mathrm{U}_{\mathrm{t}}\left(\mu_{1}, \mu_{2}\right)$ to inverse fuzzy function $\mathrm{S}_{\mathrm{t}}\left(\mu_{1}, \mu_{2}\right)$ to forecasting model $\operatorname{SFmBDR}\left(\mu_{1}, \mu_{2}\right)$ or forecasting formula $\operatorname{SFmBDR}\left(\mu_{1}, \mu_{2}\right)$. We built the forecasting model $\operatorname{SFmBDR}\left(\mu_{1}, \mu_{2}\right)$, therefore, $\operatorname{SFmBDR}\left(\mu_{1}, \mu_{2}\right)$ call as fuzzy time series forecasting model. Thus, we conclude definition 5 .

Definition 5:If have a time series forecasting problem, its universe of discourse of historical data as $N=\left\{N_{1}, N_{2}, \ldots, N_{n}\right\}$ and the universe differential rate of historical data is $\mathrm{M}=\left\{\mathrm{M}_{2}, \mathrm{M}_{3}, \ldots, \mathrm{M}_{\mathrm{n}}\right\}$. If membership $\mu_{1}$ and $\mu_{2}$ get the all figures in the interval $[0,1)$, it can get infinite fuzzy time series forecasting model SFmBDR $\left(\mu_{1}, \mu_{2}\right)$. We use whole fuzzy time series forecasting model as an element, they can form set which call SFTSFMBDR(The Set of Fuzzy Time Series Forecasting Models Based on the Difference Rate), it can abbreviate as SFmBDR. Its general element as SFmBDR $\left(\mu_{1}, \mu_{2}\right)$. SFmBDR $\left(\mu_{1}, \mu_{2}\right)$ not only express a forecasting formula of fuzzy time series, but also show the forecasting formula of fuzzy time series forecasting models.

In table1 and table2, MSE(Mean Square Error) and AFER(Average Forecasting Error Rate) can denoted as follow:

$$
M S E=\frac{1}{n} \sum_{t=1}^{n}\left(R_{t}-N_{t}\right)^{2} \quad \text { and } \quad \text { AFER }=\left(\frac{1}{n} \sum_{t=1}^{n}\left|R_{t}-N_{t}\right| / N_{t}\right) \times 100 \% .
$$

\begin{tabular}{|c|c|c|c|c|c|c|c|}
\hline Year & $\begin{array}{l}\text { Enroll- } \\
\text { ments }\end{array}$ & $\begin{array}{l}\text { Wang, } \\
\text { Guo, } \\
\text { Feng, } \\
\text { Jin.[5] }\end{array}$ & $\begin{array}{l}\text { Feng, } \\
\text { Guo, } \\
\text { Wang } \\
\text { Zhang.[6] }\end{array}$ & $\begin{array}{l}\text { Wang, } \\
\text { Guo, } \\
\text { Feng, } \\
\text { Jin.[8] }\end{array}$ & $\begin{array}{l}\text { Saxena, } \\
\text { Sharma, } \\
\text { Easo. } \\
{[9]}\end{array}$ & $\begin{array}{l}\text { Wang, } \\
\text { Guo, } \\
\text { Feng, } \\
\text { Jin.[11] }\end{array}$ & $\begin{array}{l}\text { Wang, } \\
\text { Guo, } \\
\text { Feng, } \\
\text { Jin.[12] }\end{array}$ \\
\hline 1971 & 13055 & - & - & - & - & - & - \\
\hline 1972 & 13563 & - & 13563 & - & 13486 & - & - \\
\hline 1973 & 13867 & 13745 & 13867 & 13845 & 13896 & 13813 & 13809 \\
\hline 1974 & 14696 & 14531 & 14696 & 14729 & 14698 & 14681 & 14610 \\
\hline 1975 & 15460 & 15575 & 15461 & 15412 & 15454 & 15525 & 15422 \\
\hline 1976 & 15311 & 15446 & 15312 & 15317 & 15595 & 15189 & 15299 \\
\hline 1977 & 15603 & 15555 & 15604 & 15620 & 15600 & 15685 & 15642 \\
\hline 1978 & 15861 & 15901 & 15860 & 15895 & 15844 & 15895 & 15901 \\
\hline 1979 & 16807 & 16933 & 16804 & 16786 & 16811 & 16878 & 16782 \\
\hline 1980 & 16919 & 16950 & 16920 & 16961 & 16916 & 16839 & 16935 \\
\hline 1981 & 16388 & 16601 & 16387 & 16334 & 16425 & 16505 & 16328 \\
\hline 1982 & 15433 & 15456 & 15430 & 15461 & 15657 & 15349 & 15362 \\
\hline 1983 & 15497 & 15544 & 15496 & 15497 & 15480 & 15511 & 15496 \\
\hline 1984 & 15145 & 15165 & 15143 & 15094 & 15214 & 15026 & 15077 \\
\hline 1985 & 15163 & 15187 & 15163 & 15133 & 15184 & 15051 & 15274 \\
\hline 1986 & 15984 & 15953 & 15976 & 15972 & 15995 & 15980 & 15966 \\
\hline 1987 & 16859 & 16849 & 16858 & 16805 & 16861 & 16805 & 16849 \\
\hline 1988 & 18150 & 18211 & 18150 & 18183 & 17965 & 18246 & 18312 \\
\hline 1989 & 18970 & 19077 & 18974 & 18990 & 18964 & 18926 & 18974 \\
\hline 1990 & 19328 & 19344 & 19326 & 19338 & 19329 & 19275 & 19236 \\
\hline 1991 & 19337 & 19200 & 19338 & 19346 & 19378 & 19428 & 19299 \\
\hline 1992 & 18876 & 18851 & 18872 & 18822 & 18984 & 19046 & 18951 \\
\hline $\begin{array}{l}\text { AFER } \\
(\%)\end{array}$ & & 0.462 & 0.0099 & 0.171 & 0.3406 & 0.442 & 0.273 \\
\hline MSE & & 8963 & 7 & 1121 & 9169 & 6825 & 2912 \\
\hline
\end{tabular}

TABLE I. COMPARISON OF DIFFERENT FORECASTING MODELS

Theorem1: (the Convergence theorems of fuzzy function of SFmBDR) If have a time series forecasting problem, its universe of discourse of historical data as $N=\left\{N_{1}, N_{2}, \ldots, N_{n}\right\}$ and the universe differential rate of historical data is 
$M=\left\{M_{2}, M_{3}, \ldots, M_{n}\right\}$. Thus, as every $t \in\{2,3, \ldots, n\}$, when $\mu_{1} \rightarrow 0, \mu_{2} \rightarrow 0$, the inverse function $\mathrm{S}_{\mathrm{t}}\left(\mu_{1}, \mu_{2}\right)$ restrain difference rate $\mathrm{M}_{\mathrm{t}}$ of historical data , thus:

$$
\lim _{\mu_{1} \rightarrow 0, \mu_{2} \rightarrow 0} S_{t}\left(\mu_{1}, \mu_{2}\right)=M_{t}
$$

Proof if every $t \in\{2,3, \ldots, n\}$, ordering membership $\mu_{1} \rightarrow 0, \mu_{2} \rightarrow 0$ is limited to the calculation of inverse fuzzy function (2), it can gain the calculation form as:

$$
\begin{gathered}
\lim _{\mu_{1} \rightarrow 0, \mu_{2} \rightarrow 0} S_{t}\left(\mu_{1}, \mu_{2}\right)=\lim _{\mu_{1} \rightarrow 0, \mu_{2} \rightarrow 0}\left\{\begin{array}{l}
\frac{1+\mu_{2}}{\frac{1}{M_{2}}+\frac{\mu_{2}}{M_{3}}}, t=2 \\
\frac{\mu_{1}+1}{\frac{\mu_{1}}{M_{t-1}}+\frac{1}{M_{t}}}, 3 \leq t \leq n
\end{array}\right. \\
=\left\{\begin{array}{l}
\frac{1}{\frac{1}{M_{2}}}, t=2 \\
\frac{1}{\frac{1}{M_{t}}}, 3 \leq t \leq n=\frac{1}{M_{t}}(2 \leq t \leq n)=M_{t}(2 \leq t \leq n)
\end{array}\right.
\end{gathered}
$$

Theorem 2: (the Convergence theorems of forecasting function of SFmBDR) If have a time series forecasting problem, its universe of discourse of historical data as $\mathrm{N}=\left\{\mathrm{N}_{1}, \mathrm{~N}_{2}, \ldots, \mathrm{N}_{\mathrm{n}}\right\}$ and the universe differential rate of historical data is $\mathrm{M}=\left\{\mathrm{M}_{2}, \mathrm{M}_{3}, \ldots, \mathrm{M}_{\mathrm{n}}\right\}$. Thus, as every $\mathrm{t} \in\{2,3, \ldots, \mathrm{n}\}$, when $\mu_{1} \rightarrow 0, \mu_{2} \rightarrow 0$, the forecasting function $\mathrm{R}_{\mathrm{t}}\left(\mu_{1}, \mu_{2}\right)$ restrain difference rate $\mathrm{N}_{\mathrm{t}}$ of historical data , thus:

$$
\lim _{\mu_{1} \rightarrow 0, \mu_{2} \rightarrow 0} R_{t}\left(\mu_{1}, \mu_{2}\right)=N_{t}
$$

Proof if $i \in\{2,3, \ldots, n\}$, according to formula of difference rate, knows:

$\mathrm{M}_{\mathrm{t}}=\left(\mathrm{N}_{\mathrm{t}}-\mathrm{N}_{\mathrm{t}-1}\right) / \mathrm{N}_{\mathrm{t}-1} \Rightarrow \mathrm{N}_{\mathrm{t}}=\mathrm{N}_{\mathrm{t}-1}\left(1+\mathrm{M}_{\mathrm{t}}\right)$.

Ordering membership $\mu_{1} \rightarrow 0, \mu_{2} \rightarrow 0$ is limited to the calculation of forecasting function (3) and apply to the result of theorem1, knows:

$$
\begin{aligned}
& \lim _{\mu_{1} \rightarrow 0, \mu_{2} \rightarrow 0} R_{t}\left(\mu_{1}, \mu_{2}\right)=\lim _{\mu_{1} \rightarrow 0, \mu_{2} \rightarrow 0} N_{t-1}\left(1+R_{t}\left(\mu_{1}, \mu_{2}\right)\right) \\
& =N_{t-1}\left(1+\lim _{\mu_{1} \rightarrow 0, \mu_{2} \rightarrow 0}\left(\mu_{1}, \mu_{2}\right)\right)=N_{t-1}\left(1+M_{t}\right)=N_{t}
\end{aligned}
$$

Obviously, there is Theorem3.

Theorem 3 If have a time series forecasting problem, its universe of discourse of historical data as $N=\left\{N_{1}, N_{2}, \ldots, N_{n}\right\}$ and the universe differential rate of historical data is $\mathrm{M}=\left\{\mathrm{M}_{2}, \mathrm{M}_{3}, \ldots, \mathrm{M}_{\mathrm{n}}\right\}$. If every $\mathrm{t} \in\{2,3, \ldots, \mathrm{n}\}$, thus fuzzy function $\mathrm{U}_{\mathrm{t}}\left(\mu_{1}, \mu_{2}\right)$, inverse fuzzy function and forecasting function are continuous function.

As corollary, there is theorem 4:

Theorem4 If have a time series forecasting problem, its universe of discourse of historical data as $N=\left\{N_{1}, N_{2}, \ldots, N_{n}\right\}$ and the universe differential rate of historical data is $M=\left\{M_{2}, M_{3}, \ldots, M_{n}\right\}$. If every $t \in\{2,3, \ldots, n\}$, thus, membership $\mu_{1}$ 1and $\mu_{2}$ is enough small, the forecasting function $R_{t}\left(\mu_{1}, \mu_{2}\right)$ of $\mathrm{t}$ years' SFmBDR equal to historical data $\mathrm{N}_{\mathrm{t}}$. Of t years.

In this paper, we just list sectional forecasting models and get the comparison of different forecasting methods which apply inverse fuzzy, when we forecast the problems of enrollments data of University of Alabama in 1971 1992. Show in table1 and table2. Table2 shows that use SFmBDR(0.000002, 0.000004) and SFmBDR(0.000004, 0.000002 ) forecast the results of enrollments data of University of Alabama, it can get MSE $=0$ and AFER $=0.0 \%$. That is the highest accuracy. It also verify the Theorem4 is right.

\section{CONCLUSION}

We Provide the forecasting models SFmBDR(0.000004, 0.000002)and SFmBDR(0.000002, 0.000004), and then we get $\mathrm{MSE}=0$ and $\mathrm{AFER}=0 \%$ which show in table 2 , when we study the problems of enrollments data of University of

\begin{tabular}{|c|c|c|c|c|c|c|c|}
\hline Year & $\begin{array}{l}\text { Enroll- } \\
\text { ments }\end{array}$ & $\begin{array}{l}\text { Jilani, } \\
\text { Burney, } \\
\text { Ardil. } \\
\text { [13] } \\
\end{array}$ & $\begin{array}{l}\text { Stevenson, } \\
\text { Porter. } \\
{[14]}\end{array}$ & $\begin{array}{l}\text { Wang, } \\
\text { Guo, } \\
\text { Feng } \\
\text { Zhang.[16] }\end{array}$ & $\begin{array}{l}\text { Wang, } \\
\text { Guo, } \\
\text { Wang, } \\
\text { Feng.[17] }\end{array}$ & $\begin{array}{l}\text { SFmBDR } \\
(0.000002, \\
0.000004)\end{array}$ & $\begin{array}{l}\text { SFmBDR } \\
(0.000004, \\
0.000002)\end{array}$ \\
\hline 1971 & 13055 & 13579 & - & - & - & - & - \\
\hline 1972 & 13563 & 13798 & 13410 & - & 13563 & 13563 & 13563 \\
\hline 1973 & 13867 & 13798 & 13932 & 13867 & 13867 & 13867 & 13867 \\
\hline 1974 & 14696 & 14452 & 14664 & 14691 & 14695 & 14696 & 14696 \\
\hline 1975 & 15460 & 15373 & 15423 & 15461 & 15460 & 15460 & 15460 \\
\hline 1976 & 15311 & 15373 & 15847 & 15311 & 15311 & 15311 & 15311 \\
\hline 1977 & 15603 & 15623 & 15580 & 15607 & 15603 & 15603 & 15603 \\
\hline 1978 & 15861 & 15883 & 15877 & 15861 & 15861 & 15861 & 15861 \\
\hline 1979 & 16807 & 17079 & 16773 & 16797 & 16796 & 16807 & 16807 \\
\hline 1980 & 16919 & 17079 & 16897 & 16920 & 16919 & 16919 & 16919 \\
\hline 1981 & 16388 & 16497 & 16341 & 16375 & 16388 & 16388 & 16388 \\
\hline 1982 & 15433 & 15737 & 15671 & 15436 & 15432 & 15433 & 15433 \\
\hline 1983 & 15497 & 15737 & 15507 & 15497 & 15497 & 15497 & 15497 \\
\hline 1984 & 15145 & 15024 & 15200 & 15136 & 15144 & 15145 & 15145 \\
\hline 1985 & 15163 & 15024 & 15218 & 15163 & 15163 & 15163 & 15163 \\
\hline 1986 & 15984 & 15883 & 16035 & 15861 & 15982 & 15984 & 15984 \\
\hline 1987 & 16859 & 17079 & 16903 & 16858 & 16859 & 16859 & 16859 \\
\hline 1988 & 18150 & 17991 & 17953 & 18154 & 18150 & 18150 & 18150 \\
\hline 1989 & 18970 & 18802 & 18879 & 18976 & 18970 & 18970 & 18970 \\
\hline 1990 & 19328 & 18994 & 19303 & 19329 & 19327 & 19328 & 19328 \\
\hline 1991 & 19337 & 18994 & 19432 & 19338 & 19337 & 19337 & 19337 \\
\hline 1992 & 18876 & 18916 & 18966 & 18766 & 18874 & 18876 & 18876 \\
\hline $\begin{array}{l}\text { AFER } \\
(\%)\end{array}$ & & 1.02 & 0.57 & 0.085 & 0.0026 & 0 & 0 \\
\hline MSE & & 41426 & 21575 & 1384 & 1 & 0 & 0 \\
\hline
\end{tabular}
Alabama in 1971 1992. We conclude the historical results of fuzzy time series forecasting models which accuracy is not high. We knows that sectional forecasting models of SFmBDR have important application potential.

TABLE II. COMPAROSON OF DIFFERENT FORECASTING MODELS 


\section{ACKNOWLEDGEMENTS}

Sanya science and technology cooperation project (2016YD04, 2014YD33); Hainan Provincial Natural Science Foundation (20156222, 714283)

\section{REFERENCES}

[1] Q Song, B S Chissom. Fuzzy series and its models. Fuzzy Sets and Systems, Vol. 54, pp.269-277, 1993

[2] Q Song, B S Chissom. Forecasting enrollments with fuzzy time series-Part I.Fuzzy Sets and Systems, Vol.54, pp. 1-9, 1993

[3] L A Zadeh. Fuzzy set .Fuzzy Sets and Systems, Vol. 8, pp. 338-353, 1965

[4] Q Song, B S Chissom. Forecasting enrollments with fuzzy time series-Part II.Fuzzy Sets and Systems, Vol.62, pp. 1-8, 1994

[5] Hong-Xu Wang, Jian-Chun Guo, Hao Feng, HaiLong Jin. A fuzzy time series forecasting model based on percentages. 2nd International Conference on Frontiers in Computer Education (ICFCE2014), December 24-25, 2014, Wuhan, China.1 ICT IN EDUCATION. Frontiers in Computer Education. pp: 11-14, 2014

[6] Hao Feng, Jianchun Guo, Hongxu Wang*, Fujin Zhang. A modified method of foreecasting enrollments based on fuzzy time series [C]. 2014 2nd International Conference on Soft Computing in Information Communication Technology (SCICT2014), The Authors- Published by Atlantis Press, pp. 176-179, 2014

[7] Tahseen A Jilani, S M Aqil Burney, and C Ardil. Multivariate high order fuzzy time series forecasting for car road accidents. World Academy of Science, Engineering and Technology, Vol. 1, pp: 288-293, 2007

[8] Wang Hongxu, Guo Jianchun, Feng Hao, Jin Hailong. An improved forecasting model of fuzzy time series.2014 3rdInternational Conference on Mechatronics and Control Engineering (ICMCE 2014), Applied Mechanics and Materials, Vol. 678(2014), PP: 64-69, 2014

[9] Preetika Saxena, Kalyani Sharma, Santhosh Easo. Forecasting enrollments based on fuzzy time series with higher forecast accuracy rate. Int. J. Computer Technology\& Applications, Vol.3 (3), pp, 957-961, 2012

[10] Wang Hongxu, Wu Zhenxing. Preliminary Theory of Set SDR of Fuzzy Time Series Forecasting Model. 2016 International Conference on Mathematical, Computational and Statistical Sciences and Engineering (MCSSE2016), pp:261-265, 2016

[11] Hong Xu Wang, JianChun Guo, HaoFeng, HaiLong Jin. A fuzzy time series forecasting model based on data differences. 2ndInternational Conference on Frontiers in Computer Education (ICFCE2014), December 24-25, 2014, Wuhan, China.1 ICT IN EDUCATION. Frontiers in Computer Education. pp: 15-18, 2014

[12] Wang Hongxu, GuoJianchun, Feng Hao, Jin Hailong. A new forecasting model of fuzzy time series. 2014 3rdInternational Conference on Mechatronics and Control Engineering (ICMCE 2014), Applied Mechanics and Materials, Vol. 678(2014), PP: 59-63, 2014

[13] T A Jilani, S M A Burney, C Ardil. Fuzzy metric approach for fuzzy time series forecasting based on frequency density based partitioning. Proceedings of World Academy of Science, Engineering and Technology, Vol. 34, pp, 333-338, 2007

[14] Meredith Stevenson and John E. Porter. Fuzzy time series forecasting using percentage change as the universe of discourse. Proceedings of World Academy of Science, Engineering and Technology, Vol. 55, pp, 154-157, 2009

[15] Wang Hongxu, Wu Zhenxing. Preliminary Theory of Set DR of Fuzzy Time Series Forecasting Model. 2016 International Conference on Mathematical, Computational and Statistical Sciences and Engineering (MCSSE2016), pp:256-260, 2016

[16] H X Wang, J C Guo, H Feng, F J Zhang.A new model of forecast enrollment using fuzzy time series. Education Management and Management Science, 2014 International Conference on Education Management and Management Science (ICEMMS 2014), 7-8 August,2014,Tianjin, China, pp: 95-98, 2014
[17] Hongxu Wang, JianchunGuo, Hui Wang, HaoFeng*. A fuzzy time series forecasting model based on yearly difference of the student enrollment number [C]. 2014 2nd International Conference on Soft Computing in Information Communication Technology (SCICT2014), The Authors- Published by Atlantis Press, pp. 172-175, 2014 